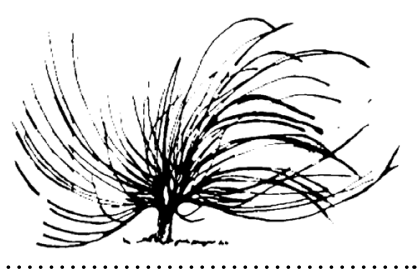

\title{
Praxis pedagógica desde la inclusividad: Una mirada a la docencia universitaria de la Universidad Nacional de Costa Rica
}

\author{
Stephanie Valdivia Durán ${ }^{1}$ \\ Ministerio de Educación Pública \\ Costa Rica \\ tefa0893-e@hotmail.com
}

\begin{abstract}
Resumen
Hoy, las prácticas educativas inclusivas son un reto en Costa Rica, específicamente, en el área educativa hay muchos aspectos por mejorar. Esta situación no es ajena a la educación superior, por ello, en este espacio se pretende exponer cómo se viven las prácticas educativas en la praxis de la docencia universitaria de la Universidad Nacional. Esto se hará con el objetivo de conocer cuáles han sido y siguen siendo los avances y desafíos que se deben atender desde la inmediatez. A raíz de esto, se contextualiza sobre la realidad del país en cuanto a la población con discapacidad, se realiza una aclaración conceptual e histórica de la inclusión y se exponen la legislación que ampara a esta población, los avances y desafíos a los que se enfrenta la docencia de la Universidad Nacional en materia de inclusión y una serie de propuestas sobre las que se podría basar la universidad para enfrentar los retos encontrados.
\end{abstract}

Palabras clave: Docencia universitaria, prácticas educativas inclusivas, praxis pedagógica, Universidad Nacional.

http://dx.doi.org/10.15359/rep.esp-21.10

1 Docente de secundaria con el Ministerio de Educación Pública y en el sector privado. Miembro del Colectivo A de Libertad, de la Editorial Ediciones Libres y del Centro de Amigos para la Paz. Bachiller en la Enseñanza de los Estudios Sociales y la Educación Cívica en la Universidad Nacional. Licenciada en Pedagogía con Énfasis en Didáctica en la Universidad Nacional. Actualmente cursa la Maestría en Educación con Énfasis en Pedagogía Universitaria en la Universidad Nacional. 


\begin{abstract}
Today, inclusive educational practices are currently a challenge in Costa Rica; specifically, in the area of education, there are many aspects that need to be improved. This situation is not unrelated to higher education, so in this paper is intended to approach how educational practices are lived in the actual practice of university teaching at Universidad Nacional, Costa Rica. This will be done with the aim of knowing what progress has been made and what challenges still need to be addressed immediately. As a result, the reality of the population with disabilities is contextualized; a conceptual and historical clarification about inclusion is made; and the legislation protecting this population, the scope, and challenges in terms of inclusion faced by faculty at Universidad Nacional are described along with a series of proposals that the university could consider to face the challenges encountered.
\end{abstract}

Keywords: University teaching, inclusive educational oractices, pedagogical practice, Universidad Nacional.

"Por un mundo donde seamos socialmente iguales humanamente diferentes y totalmente libres" Rosa Luxemburgo

\title{
Introducción
}

L

a labor docente implica el constante contacto con la diversidad; por tanto, es deber del personal docente considerar este aspecto en su trabajo. Se debe comprender que la diversidad abarca muchos sectores poblacionales. Por ello, en este ensayo se pretende exponer una reflexión sobre la praxis pedagógica a nivel de educación superior y desde un enfoque que contemple las diferentes necesidades del estudiantado.

Referente a lo anterior, en este ensayo, la diversidad se dirige, específicamente, al abordaje pedagógico con las personas que presentan alguna deficiencia de índole sensorial, psicosocial, física o cognitiva. La razón del enfoque en esta población radica en que, para el caso de Costa Rica, la educación inclusiva para personas con alguna deficiencia 
sigue siendo un reto, tal como lo revelan los datos del Instituto Nacional de Estadística y Censo (INEC), evidenciados en este ensayo.

Este texto tuvo origen en la reflexión sobre la experiencia propia, por esta razón, la docencia y la formación que se ha vivido en la Universidad Nacional de Costa Rica son el contexto de referencia , específicamente, el área de formación de formadores; no obstante, no se descarta la posibilidad de que esta realidad se viva también en otros centros de educación superior. En este proceso de autorreflexión se encontró que, en las distintas etapas de formación, no se lograron adquirir conocimientos sobre las prácticas educativas inclusivas, por ello, el objetivo de este ensayo es manifestar la necesidad e importancia de este tipo de saberes en la docencia.

A raíz de esto, fue necesario consultar y contrastar con la realidad algunos documentos que muestran la legislación existente en el ámbito nacional y que busca amparar la igualdad de oportunidades para la población con discapacidad. Asimismo, se consultaron textos propiamente de la Universidad Nacional, tal como su Modelo Pedagógico, las políticas institucionales y los lineamientos curriculares. De igual forma, se pretenden aclarar algunos aspectos conceptuales e históricos sobre el tema de la inclusión.

La revisión de este tipo de documentos tiene como fin exponer los logros obtenidos en materia de inclusión y, asimismo, evidenciar los desafíos que no se han podido solventar. De igual forma, con base a este panorama, se busca visualizar posibles soluciones hacia donde se podría encaminar la universidad, utilizando los recursos existentes de manera textual, pero que no siempre se logran aplicar en la realidad.

Para abordar esta reflexión, el ensayo se compone de cinco partes, además de un espacio para conclusiones. El primer apartado consistirá en una contextualización de la realidad de Costa Rica en cuanto a la población con discapacidad y la importancia de la reflexión de la práctica pedagógica universitaria. La segunda temática se centra en la inclusión y los paradigmas bajo los cuales se está comprendiendo. En el tercer espacio, se describe la legislación a nivel nacional, la que ampara a esta población. En el siguiente apartado, se describen las prácticas educativas inclusivas y se comentan las labores de la Universidad Nacional para ejecutarlas a nivel institucional. La quinta parte, se destinó a exponer los aspectos que podría considerar la Universidad Nacional para fortalecer la formación de la docencia universitaria. Por último, se cierra con el espacio de las conclusiones. 


\section{Discapacidad en Costa Rica y reflexión de la praxis pedagógica universitaria}

Tal y como se mencionó al inicio de este trabajo, el abordaje de la educación para las personas con discapacidad en el país sigue representando un reto. Esta realidad debe atenderse con inmediatez, pues la cantidad de personas que están en dicha condición es significativa. Según la Encuesta Nacional sobre Discapacidad (Enadis) del 2018, el $18,2 \%$ de personas de 18 años y más, que habitan en el país, presenta una discapacidad, este porcentaje representa 670640 personas, aproximadamente. Además, se revela que de ese $18,2 \%$, un $14,4 \%$ la posee en un grado severo y solo un 3,8 \% la tiene leve o moderada.

Antes de comentar la información expuesta, es necesario distinguir la deficiencia de la discapacidad, pues según explica Madrigal (2015), la deficiencia es una característica de la persona, mientras que la discapacidad es la condición provocada por el entorno y por las actitudes de las personas. Por ejemplo, una deficiencia puede ser experimentada por alguien con limitación visual; sin embargo, lo que la hace discapacitada es encontrarse en un entorno que no le genere las condiciones para desenvolverse de la misma manera que una persona que no posea tal deficiencia.

Por ello, los datos expuestos en el Enadis reflejan que la mayoría de la población con discapacidad se vio condicionada por barreras impuestas por el entorno, esto dificultó su desarrollo y les generó la discapacidad; pues si el contexto estuviese brindando las herramientas necesarias para estas personas, no se tendrían mayores porcentajes de discapacidad severa con respecto a la leve o moderada.

Referente a lo anterior, existen muchas características en el entorno que pueden generar discapacidad, tal como el acceso a la salud, al trabajo, a una educación de calidad, entre otras. Para el caso de la educación, es necesario realizar procesos de investigación y reflexión sobre la praxis de la docencia, pues tal y como menciona López (2018), "por eso construimos nuevas teorías a partir de la práctica y de la reflexión de la misma" (p. 21). Este tipo de investigaciones promueven que la docencia genere conocimiento sobre las exigencias requeridas para su formación. Sin embargo, según Vijil (2011), es muy poco el intercambio de experiencias entre docentes y programas de actualización; esto refleja que los procesos de sistematizar y reflexionar sobre la praxis no son una constante en la docencia. 
No obstante, una de las causas por las que la docencia no siempre sistematiza sus experiencias es por el poco espacio disponible. Con el pasar de los años, se le atribuyen más tareas a la labor docente, por lo que el tiempo y el recurso para investigar es cada vez menor. Además, a raíz de las decisiones políticas y económicas, surgen más "docentes llamados 'taxis', porque dan clases en tres o cuatro universidades, sin tiempo para leer, ponerse al día y planear sus clases" (Vijil, 2011, p. 14).

Con base a lo anterior, se puede ver que es una necesidad que la docencia reflexione sobre su propia praxis, pero también se requieren decisiones a nivel de las universidades para generar espacios donde la docencia pueda investigar y escribir sobre su propia praxis. Es por esto que este ensayo pretende mostrar el resultado de una reflexión sobre el quehacer docente universitario de la Universidad Nacional, donde se descubrió la necesidad de una formación desde la inclusión.

\section{¿A qué se le llama inclusión?}

Para tener una docencia universitaria formada desde la inclusión es necesario conocer su significado. Según Álvarez (2012), "el término inclusión pone el acento en cómo construir un sistema que incluya y que esté estructurado para satisfacer las necesidades de cada uno" (p. 28). Esto implica que el entorno y las actitudes de las personas integrantes del medio, modifiquen sus acciones de manera en la que se puedan hacer inclusiones sin importar características de estos individuos.

Si se une la idea anterior con el ámbito educativo, crear un espacio inclusivo implicaría no solo que el sistema sea flexible a nivel curricular, sino que exista material didáctico, metodologías de mediación pedagógica, población estudiantil y docentes inclusivos, pues solo así se puede promover el desarrollo integral y exitoso de todo el estudiantado.

En este sentido, la inclusión en el área educativa se refiere, según Echeita et al. (2011), a que todo el alumnado tenga posibilidades de estar presente, participar y rendir. Esto quiere decir que una educación de este tipo debe crear las condiciones adecuadas para las necesidades de las personas que coexisten en una misma aula.

Este tipo de ambiente apela a toda la diversidad que pueda existir dentro de un salón de clases, puede ser en cuanto a género, condición socioeconómica, creencia religiosa, orientación sexual, etnia, entre otras. Sin embargo, como se mencionó al inicio del ensayo, en esta ocasión se delimitará a la inclusión de personas con deficiencias, cuyo 
entorno les hace vivir con discapacidad, por lo que poseen necesidades educativas específicas.

Para ello es necesario comprender en cuál paradigma se encuentra la inclusión de esta población en el área educativa, pues se han delimitado tres diferentes teorías que han ido evolucionando. A partir de ellas se les ha comprendido: el paradigma tradicional o de prescindencia, el paradigma clínico rehabilitador y el paradigma social.

\section{Paradigma tradicional o de prescindencia}

Bajo este paradigma, se concebía a las personas con discapacidad como víctimas del castigo de las divinidades y como personas que no podían aportar nada a la comunidad. A raíz de esto, según Velarde (2012), surgieron dos submodelos: el eugenésico, con el que se eliminaba la vida del niño que naciera con una discapacidad y el modelo de marginación, con el cual se alejaba al menor de la sociedad.

Además, según esta misma autora, las personas con discapacidad que lograban sobrevivir, por lo general fueron objeto de burla y entretenimiento. Caso contrario sucedía con las personas que nacían sin discapacidad, pero adquirían una, pues estas personas más bien cobraban pensiones; tal era el caso de los soldados heridos en guerra.

\section{Paradigma clínico rehabilitador}

Este modelo viene a sustituir el tradicional. Con este ya no se piensa que la discapacidad es producto de un castigo divino, sino que más bien existe una razón científica. Según Velarde (2012), "las personas con discapacidad dejan de ser consideradas inútiles respecto de las necesidades de la comunidad y, siempre que sean rehabilitadas, pueden tener algo que aportar" (p. 123).

Por esta razón, este paradigma busca "curar" a las personas con alguna discapacidad, ya sea erradicando el supuesto problema o modificando las conductas para esconder las diferencias de la persona, de manera que se logre incorporar en la sociedad. Es decir, para este paradigma, una persona con discapacidad "requiere de cuidados médicos proporcionados por profesionales bajo formas de tratamientos individuales" (Velarde, 2012, p. 125).

Es necesario reconocer que en la actualidad la sociedad aún se encuentra en una transición de este paradigma hacia el paradigma social, pues aún es común escuchar a personas cuestionando cómo 
pueden curar a alguien con alguna condición como el autismo, y cualquier especie de discapacidad a nivel de los cinco sentidos. Con este se ve que, a nivel social, aún no se conciben estas características como parte de la diversidad, sino más bien como un "problema" que hay que regular y, en la medida de lo posible, curar. Otro ejemplo, de esta transición es la reciente firma del decreto 40955. En ella se estipula que las aulas diferenciadas se deben cerrar para que el estudiantado con discapacidad sea parte de las aulas ordinarias. El rezago se da tras una demora porque, a pesar de que la legislación fue creada años atrás, la medida se tomó en 2018.

\section{Paradigma social}

Este es el paradigma en el que se basan las prácticas educativas inclusivas, pues en vez de comprender la discapacidad como una carencia de la persona que debe ser curada para que se pueda incorporar en la sociedad, más bien, se busca que sean la sociedad y el entorno los que se adapten a las necesidades de todas las personas.

Justamente, desde este modelo surge la diferencia entre la deficiencia y la discapacidad explicada al inicio de este ensayo. En esta diferencia se comprende que "las causas que originan la discapacidad no son ni religiosas ni científicas, sino sociales" (Velarde, 2012, p. 130); por lo que el problema no sería las deficiencias de las personas, sino la incapacidad de la sociedad para permitir a la comunidad ser parte de la organización social.

A pesar de que ya se hable de este paradigma, la sociedad aún sigue con conductas propias de la visión clínico-rehabilitadora. Las herramientas para pasar al paradigma social sí tienen un apoyo de la legislación, pues se han logrado avances desde el plano legal; sin embargo, las actitudes de la sociedad en general no son acordes al pensamiento del paradigma social, porque todavía se escuchan expresiones como "personas con problemas, son especiales, pobrecitos".Todos estos usos no muestran una concientización de la diversidad.

Por ello, es necesario brindar procesos de aprendizaje sobre el tema en la sociedad; para lograrlo existen muchas acciones por considerar, entre ellas, convivir con personas de diferentes condiciones para perder el temor o prejuicio de sus realidades, desarrollar la empatía y utilizar la legislación para llevar a cabo proyectos inclusivos y educativos. 


\section{Legislación nacional en materia de inclusión}

Como se mencionó, se han logrado avances en cuanto a la legislación que contribuye a una adecuada inclusión; sin embargo, en muchos casos, estas leyes no se cumplen a cabalidad, pues si se respetaran no se estarían viendo los porcentajes tan elevados de personas con discapacidad severa, expuestos al inicio del ensayo.

Por esta razón, en este espacio se demuestran los planteamientos de los mecanismos legales en materia de educación. Para ello, se alude a la Política Nacional en Discapacidad, la Ley 7948, Ley 8661 y la Ley 7600. Cada una de ellas incluye diferentes aspectos para construir una sociedad inclusiva, entre esos aspectos se rescata el área de educación en los diferentes niveles de formación existentes en Costa Rica. No obstante, para efectos del enfoque de este ensayo, se recurre únicamente a las que responden al nivel de Educación Superior.

El primer marco político a mencionar es la Política Nacional en Discapacidad (PONADIS) 2011-2021; esta instituye la dirección que debe seguir el Estado Costarricense, durante diez años (en este caso se expone la del periodo 2011-2021), para garantizar un adecuado desarrollo y respeto por las personas con discapacidad, de manera que el PONADIS expone las directrices para la "ejecución de lineamientos y estrategias que han de ser desarrollados por la institucionalidad pública en el periodo 2010-2021, para lograr el cierre de brechas de inequidad que inciden en el desarrollo inclusivo de la población con discapacidad" (2012, p. 16).

Estas directrices se basan en diferentes ámbitos como salud, educación, empleo, institucionalidad, entre otras. En el área de educación, se habla de manera general, al mencionar al Ministerio de Educación Pública y a la formación de los niños, niñas y adolescentes. Sin embargo, no se muestra el caso concreto de la Educación Superior. A pesar de esto, el documento sí se puede encontrar en la página de la Universidad Nacional.

Otro marco jurídico es la Convención Interamericana Para la Eliminación de Todas Las Formas De Discriminación Contra Las Personas Con Discapacidad (Ley 7948); esta contempla las diversas formas en las que se puede discriminar a una persona con discapacidad, por lo que también vela por el derecho a la igualdad de trato. Uno de los artículos rescatados de esta Ley es el Artículo III. En él se estipula que se deben adoptar todas las medidas necesarias para eliminar la discriminación 
contra las personas con discapacidad en materia legislativa, social, educativa, laboral o de cualquier índole que permita una adecuada integración de la persona en la sociedad.

En cuanto a la Convención Sobre Los Derechos De las Personas Con Discapacidad (Ley 8661), esta también busca garantizar las condiciones necesarias para todas las personas con discapacidad, estas "incluyen a aquellas que tengan deficiencias físicas, mentales, intelectuales o sensoriales a largo plazo que, al interactuar con diversas barreras, puedan impedir su participación plena y efectiva en la sociedad, en igualdad de condiciones con las demás" (p. 4).

Para ello, al área de educación se le destina el Artículo 24, donde se asegura que el Estado debe reconocer el derecho de las personas con discapacidad a recibir educación bajo igualdad de oportunidades. Esto implica que el acceso sea en todos los niveles de enseñanza. En este mismo artículo se menciona que el Estado debe asegurar que las personas con discapacidad "tengan acceso general a la educación superior, la formación profesional, la educación para adultos y el aprendizaje durante toda la vida sin discriminación y en igualdad de condiciones con las demás" (p. 20).

Por último, la Ley De Igualdad De Oportunidades Para Las Personas Con Discapacidad (Ley 7600), en los títulos II y III, contiene aspectos relativos a la educación. El Artículo 14 especifica que el Estado debe garantizar el acceso a la educación para todas las personas indistintamente de la discapacidad que se posea; esto implica desde la estimulación temprana hasta la educación superior, tanto a nivel público como privado.

Para garantizar esto, el Artículo 17 de esta misma ley expresa que los espacios educativos deben de realizar las adaptaciones necesarias y brindar los apoyos requeridos para hacer valer el derecho a la educación de la ciudadanía con discapacidad. Estas adaptaciones y apoyos implican que se realice desde "los recursos humanos especializados, adecuaciones curriculares, evaluaciones, metodología, recursos didácticos y planta física" (p. 7).

En cuanto a las acciones desde la educación superior propiamente, se asegura en el Artículo 57 que el Estado promoverá y apoyará que se "impartan carreras de formación específica en todas las disciplinas y niveles, a fin de que la equiparación de oportunidades de las personas con discapacidad esté efectivamente garantizada" (p. 17). Para ello, 
según el Artículo 58, a los centros de educación superior les corresponde tomar en cuenta contenidos sobre la discapacidad en todas las áreas de formación, es decir que debe estar inmerso en el currículo de todas las carreras y niveles.

Estas legislaciones mencionadas exponen, en general, la necesidad de garantizar la igualdad de oportunidades para las personas con discapacidad, con el fin de que se puedan desarrollar de manera integral en la sociedad. Y como se analizó, un elemento fundamental es la educación; con esto se logró demostrar que, de las cuatro legislaciones destacadas, dos hablan de manera general en cuanto a la educación y la otra mitad sí contiene artículos en los que se especifica sobre la Educación Superior, principalmente, la Ley 7600. En ella se puntualiza más sobre las garantías, para las personas con discapacidad, que le compete cumplir al Estado en el contexto universitario.

La normativa es clara: la formación de las futuras personas profesionales del país debe estar enfocada en la inclusividad. No obstante, la realidad es que estas legislaciones tienen muchos años en vigencia y, aun así, no se están logrando cumplir a cabalidad. Es necesario que, además de incorporar la educación primaria y secundaria en la legislación, se desarrollen de manera más específica las oportunidades que se deben garantizar en la Educación Superior.

Asimismo, es fundamental promover un mayor sentido de concientización, pues si bien es cierto, la legislación puede mejorar, tampoco es un área que se encuentre rezagada, hay suficientes avances en materia jurídica, ellos resguardan que en las instituciones se esté cumpliendo con la inclusión como aspecto intrínseco en la formación de un futuro profesional. Lo que aún tiene que asimilarse es la concientización y el desarrollo de habilidades para una formación inclusiva, pues solo de esta manera se logrará cumplir con lo estipulado por la ley y no solo porque sea una ley, sino por empatía hacia la población con alguna discapacidad, eso llevaría a realizar en el caso de la docencia, prácticas educativas inclusivas.

\section{Las prácticas educativas inclusivas en la Universidad Nacional}

Las prácticas educativas inclusivas aún son un reto en el contexto universitario y para poder hacer frente a esto también es necesario comprender a qué se le llama "práctica educativa inclusiva", pues si bien es cierto que es necesario atender a la población universitaria con 
discapacidad, también se deben responder las necesidades del estudiantado que no posee una. En este sentido, para Álvarez (2012) la atención a la población universitaria con discapacidad no consiste únicamente en garantizar un acceso igualitario y en prácticas académico-curriculares, "sino que también se deben implantar programas especializados que potencien el desarrollo de estos alumnos/as en el plano social, personal, académico y profesional" (p. 15).

De ninguna manera, esto debe significar la desatención a otros sectores poblacionales universitarios, pues tal como lo plantean Moliner et al. (2017):

... la inclusión implica un giro radical en la idea de ayudar solo a los estudiantes con discapacidades o con n.e.a.e [necesidades educativas académicas especiales]. El interés se centra ahora en el apoyo a las necesidades de cada miembro de la escuela, independientemente de sus características. (p. 14)

Referente a lo anterior, una práctica inclusiva puede ser una misma estrategia metodológica que se pueda aplicar a toda la población estudiantil, sin importar cuál sea su condición, únicamente, debe ser lo suficientemente flexible para poder modificar su abordaje didáctico.

Por ejemplo, si el personal docente decide que el estudiante elabore un ensayo, el mismo debe adaptarse a la condición del estudiantado, pues habrá estudiantes que por su condición aún no logren hacer grandes extensiones y habrá otros estudiantes que más bien logren hacer ensayos con alto nivel de complejidad, pero al final toda la comunidad participante realizaron el ejercicio de escribir. De esta manera, se estaría logrando que la enseñanza universitaria fuera inclusiva, pues contemplaría la heterogeneidad de la población estudiantil, respondiendo a la diversidad y a las necesidades de cada estudiante.

En el caso de la Universidad Nacional, existen avances en materia de inclusión, algunos han sido las políticas universitarias que contribuyen a la parte del amparo legal; asimismo, existen programas y/o experiencias que podrían ser un portillo hacia la inclusión. No obstante, aún se cuenta con ciertas carencias. Por ello, en este espacio se refieren los avances y carencias vividas en el ámbito universitario, de manera que en el próximo apartado se expongan posibles soluciones por las cuales podría optar la Universidad para enfrentar las necesidades sociales en materia de educación inclusiva. 
Dentro de las políticas institucionales de la Universidad Nacional existen distintos enfoques, en este caso hay tres en específico que responden a la temática abordada en este ensayo. Con respecto a la actividad académica, se afirma que la Universidad "Coadyuva a la promoción de los sectores sociales menos favorecidos, mediante la puesta en práctica de políticas de inclusión e igualdad de oportunidades" (Consejo Universitario, 2016, p. 1). Esta política institucional alude a una universidad que responde a la inclusión, pues abarca todo tipo de comunidades. No obstante, esto aplica principalmente al enfoque de acceso, no especifica en cuanto a prácticas educativas y recurso humano.

Respecto a la formación de profesionales, las políticas institucionales afirman que la Universidad Nacional "garantiza la formación de profesionales capaces de interpretar y dar respuesta a los retos de las tendencias actuales del desarrollo con un alto compromiso social, respeto a la diversidad y promoción de la equidad" (Consejo Universitario, 2016, p. 2). Con esta afirmación se garantiza que, en su formación, los profesionales de la Universidad deben estar capacitados para responder a las necesidades de la sociedad, esto implica que el personal docente que imparte clases en la Universidad tenga la formación necesaria para potenciar esta característica en las diferentes carreras.

Esto último, en cuanto al reto social de la inclusión, se está cumpliendo en la Universidad, al menos en el aspecto legal, coincidiendo con el Artículo 58 de la Ley 7600, como se mencionó, estipula que los centros de educación superior deben tomar en cuenta contenidos sobre la discapacidad en todas las carreras y niveles.

Lo anterior revela que la universidad ha hecho esfuerzos por contemplar este tema, un ejemplo de ello es que los profesionales que se desenvuelven en educación especial, preescolar y educación general básica, consideran, en sus planes de estudios, cursos específicos para desarrollar habilidades necesarias para trabajar con personas con discapacidad. Sin embargo, existen otros planes de estudios de carreras de enseñanza de diferentes áreas y niveles, en los cuales se podría considerar incluir cursos de este tipo, pues solo cuentan con uno en el que se habla de diversidad, mas no se enfatiza en la población con alguna necesidad específica.

Lo anterior ha sido tema de discusión nacional después de que en febrero del 2018 se aprobara en Costa Rica el decreto 40955, en el cual se estipula el acceso de las personas con discapacidad en el sistema 
educativo. Con ello, el Ministerio de Educación Pública de Costa Rica se ve condicionado a dar cierre a las aulas diferenciadas y a brindar las líneas de acción, con las cuales en los centros educativos habrá un docente de Educación Especial; sin embargo, no implica que habrá un especialista para cada estudiante con alguna condición. Esto quiere decir que todo el cuerpo docente debe saber cómo trabajar con las distintas características de la población estudiantil, con o sin discapacidad.

La discusión de este tema ha sido una constante entre el personal docente de educación secundaria; sin embargo, para la Universidad Nacional también es de interés, pues implica que, a nivel curricular la Universidad tendrá que hacer los ajustes necesarios para capacitar a educadores y educadoras. Asimismo, a nivel de la comunidad profesional en educación que han egresado, se les pueden ofrecer cursos de actualización profesional. No obstante, este tipo de medidas se podrán tomar en cuenta siempre que los formadores de formadores también sean capacitados para abordar este tipo de población.

Aunado a este reto, se debe mencionar que en la universidad también existen profesionales con la capacidad de atender a la población con discapacidad desde un enfoque inclusivo que integre a toda la población. Este tipo de capacitaciones serán posibles siempre que se ofrezcan los recursos necesarios; un ejemplo de un recurso imprescindible, en la actualidad, es el tecnológico, pues sobre esto la Política Institucional afirma que la Universidad "garantiza la adquisición de recursos tecnológicos que favorezcan el desempeño académico y la laboral de las personas con discapacidad" (Consejo Universitario, 2016, p. 5). En este sentido, es necesario cumplir este tipo de demandas, de otra manera no se estaría correspondiendo a las políticas institucionales ni al Modelo Pedagógico, el cual será abordado en otro apartado.

Lo descrito tiene que ver con lo que Álvarez (2012) menciona como un reto de práctica real donde haya concordancia:

desde la gestión de las políticas universitarias al aula concreta de una materia específica, en la cual se realicen las adaptaciones y ajustes pertinentes para que la enseñanza superior sea realmente para todos, sin merma alguna de su calidad y excelencia. (p. 39)

Ahora bien, también existen esfuerzos de práctica real que la universidad ha logrado, uno de ellos es un programa de aprendizaje 
del Lenguaje de Señas Costarricense (LESCO). Este es ofrecido a la población estudiantil. Asimismo, en el Centro de Investigación y Docencia en Educación existen programas que promueven la inclusión; no obstante, es necesario visibilizarlos más ante la población, pues para efectos de este ensayo no se pudo tener acceso a ellos.

\section{¿Hacia dónde se puede ir como universidad en el ámbito de la inclusión?}

La Universidad Nacional no es solo un portillo hacia la inserción laboral, es en mayor medida el espacio donde una persona profesional tiene la oportunidad de desarrollar un pensamiento crítico, humanista, creador, transformador y de conocimiento no solo en su área, sino de la realidad social a nivel nacional e internacional. Es en esto en lo que radica la importancia de que sea una universidad inclusiva, pues si el único fin fuera formar mano de obra trabajadora, existirían muchos otros lugares donde aprender un oficio. No obstante, son pocos los espacios donde una persona puede tener un desarrollo educativo tan integral. De aquí que la integración del estudiantado con discapacidad en el contexto universitario debería ser un reto primordial, pues cuando no logran vivir la oportunidad de estar en la Universidad Nacional esta población pierde muchos beneficios.

Ante esta realidad, tal como lo plantea Álvarez (2012):

... el primer reto para la enseñanza superior es el de asumir que promover la inclusión no implica renunciar a la calidad y a la excelencia en el ámbito universitario sino que, todo lo contrario, es la fórmula más adecuada para promoverlas y garantizarlas. (p. 38)

Esto es fundamental rescatarlo, pues la idea de realizar prácticas educativas inclusivas no es disminuir el nivel de exigencia para la población con discapacidad, más bien, el objetivo es llevar la formación de profesionales y toda la población estudiantil de la Universidad Nacional a su máximo nivel de desarrollo.

Modelo pedagógico, actualización profesional, renovación curricular y apoyo a los recursos tecnológicos

La Universidad Nacional no puede ni debe dejar de responder a su modelo pedagógico, en el cual se manifiesta que el objetivo final de la universidad es buscar siempre el desarrollo de la autonomía como 
una forma de educar para la vida. Para ello, este modelo busca la formación integral y la educación permanente de la comunidad estudiantil, con el fin de que este actúe de manera responsable para construir una sociedad justa y solidaria.

Por esta razón, el modelo pedagógico de la Universidad parte de principios como: "respeto a la diversidad en todas sus expresiones. Respeto y compromiso con la igualdad de oportunidades y con la construcción de una sociedad más justa y equitativa. Formación de profesionales solidarios y comprometidos con el bienestar social" (Universidad Nacional, 2012, p. 5).

Asimismo, y coincidiendo con las políticas institucionales, el modelo afirma que desde el momento cuando se fundó la Universidad Nacional, se ha comprometido con el ideal de una sociedad justa, equitativa y libre, donde exista igualdad de oportunidades y el desarrollo integral de todos los sectores de la sociedad. Esto implica que la docencia universitaria tenga la formación y los recursos necesarios para desarrollar prácticas educativas inclusivas.

Coincidiendo de nuevo con las políticas institucionales, este modelo manifiesta dos ideas de la enseñanza y aprendizaje sobre el quehacer docente. En cuanto los procesos de enseñanza y aprendizaje se afirma que:

En los diversos espacios de enseñanza y aprendizaje (aulas, laboratorios, gimnasios, aulas virtuales, bibliotecas, entre otros) confluyen personas con diversos estilos, con experiencias propias de sus contextos, con diferentes capacidades, lo que requiere variedad en los procesos de mediación pedagógica y la utilización de diversas estrategias. (Universidad Nacional, 2012, p. 8)

Referente al quehacer universitario, se menciona que "la docencia universitaria presupone: La actualización permanente y el desarrollo profesional continuo como vehículos para promover la calidad académica en todas sus formas. Entornos de aprendizaje alternativos que se apoyan en las nuevas tecnologías" (Universidad Nacional, 2012, p. 7).

En este sentido, la universidad debe direccionarse hacia procesos de actualización profesional en la docencia universitaria, esto con el objetivo de que logre cumplir con lo estipula la legislación nacional, las políticas institucionales y el Modelo Pedagógico de la Universidad 
Nacional, en materia de inclusión al tiempo que logre realizar prácticas educativas inclusivas más que integradoras.

Estos procesos de actualización profesional no son una alternativa que sea imposible, ya que actualmente el personal de la universidad tiene acceso a cursos de actualización profesional. Además, esta idea de la actualización profesional se encuentra amparada por los lineamientos de flexibilidad curricular de la Universidad Nacional, pues en estos se menciona la "apertura de la oferta académica mediante programas de educación continua y actualización. Esta formación se visualiza como un proceso continuo y permanente que permite atender las necesidades educativas emergentes de graduados, egresados, estudiantes y docentes" (Consejo Universitario, 2012, p. 6).

En este sentido, se podrían brindar cursos específicos para desarrollar habilidades que permitan al personal docente actuar desde la inclusión, o bien, incorporar este tema de manera intrínseca en las temáticas de otros cursos. Asimismo, es necesario que el cuerpo docente de la universidad tenga formación pedagógica, pues existe mucho docente universitario especialista en su área de conocimiento, pero no tienen el complemento pedagógico. Esto plantea un problema, pues tal y como lo plantea Vijil (2011):

El hecho que los docentes universitarios en general no tienen formación pedagógica hace que muchas veces no se planifiquen las clases, no se conozcan métodos alternativos para la docencia y para el trabajo de los estudiantes y, sobre todo, no se dé suficiente énfasis a la promoción de los aprendizajes de los estudiantes. (p. 15)

Por esta razón, la universidad debe paralela con la formación pedagógica de toda la docencia universitaria y, de manera intrínseca promover las prácticas educativas inclusivas, pues solo con el recurso humano, la universidad podrá garantizar la igualdad de oportunidades con todo tipo de población, incluyendo a la ciudadanía con discapacidad.

Otro rumbo al que se debe encaminar la Universidad es a la renovación curricular, pues la actualización profesional es una opción para quienes ya son egresados de la universidad o quienes están ejerciendo en ella y no son egresados de la misma. Por otro lado, la renovación curricular podría ayudar a solventar que las futuras generaciones ya no 
vean la inclusión como un reto, sino como una realidad que pueden asumir sin ningún problema.

Esta posibilidad es accesible, pues la Universidad Nacional cuenta con un currículum que permite la realización de cambios necesarios. En este sentido, según los lineamientos para la flexibilidad curricular de la Universidad Nacional, el objetivo de tener un currículum flexible es que:

[...] promueve la valoración crítica del quehacer universitario, ofrece la posibilidad de responder a los retos que derivan de una sociedad en continuo cambio, la cual exige actuar frente a las demandas de los actores sociales y del entorno mismo a fin de ofrecer propuestas innovadoras en la formación de profesionales. (Consejo Universitario, 2012, p. 1)

En este sentido se ve que, en cuanto a gestión, realizar actualizaciones curriculares es posible. Es claro que realizar esto es un gran esfuerzo que requiere mucho tiempo y dedicación de quienes coordinan cada una de las unidades de la universidad; sin embargo, es necesario realizar estos procesos, pues la necesidad de la inclusión es cada vez mayor, no se puede seguir posponiendo este tema con la justificación de que implica mucho trabajo, pues entre más pase el tiempo, más complejo se puede tornar el proceso.

Realizar este tipo de renovaciones ni siquiera tiene que significar hacer labores que impliquen muchos años para poder ejecutarse, existen algunos rubros que favorecen la flexibilidad curricular y que también están contemplados en los lineamientos de la flexibilidad curricular de la Universidad Nacional. Uno de estos aspectos tienen que ver con la incorporación de ejes transversales institucionales, entre los que se incluyen conocimientos, actitudes, comportamientos, principios, valores y concepciones congruentes con la misión, visión y valores de la Universidad Nacional. Según dichos lineamientos, estos ejes deben estar presentes a lo largo de las carreras y en los contenidos. Es decir, que incluso sin tener que hacer grandes cambios a nivel curricular, se podrían potenciar los ejes de manera intrínseca en cada carrera.

Cada unidad académica debería garantizar que tanto la población que se va a egresar como la docencia que forma a estos futuros profesionales estén cumpliendo con un currículo que responda a los principios de equidad, participación plena e igualdad de oportunidades, pues en 
los lineamientos de la flexibilidad curricular se vuelve a reiterar en lo que estipula la Ley 7600 en cuanto a garantizar el acceso a la educación para la población con discapacidad, resguardando "la accesibilidad, atención y respeto a la diversidad" (Consejo Universitario, 2012, p. 6).

Para garantizar esto, una buena opción es redireccionar apoyo a los equipos tecnológicos, pues el contexto universitario no debería olvidar la necesidad de utilizar estos recursos, principalmente si se buscan las prácticas educativas inclusivas pues, tal como lo plantea Álvarez (2012), "las tecnologías de la información (TIC) pueden llegar a ser imprescindibles para el proceso de enseñanza-aprendizaje del alumnado con necesidades educativas específicas" (p. 67).

El uso de los recursos tecnológicos puede ayudar a algunas poblaciones con deficiencias a superar obstáculos específicos, además de que, en general, a cualquier estudiante se le podría conectar con diferentes entornos virtuales, a diferentes fuentes de información y a desarrollar habilidades que serán de utilidad cuando tengan que integrarse a un entorno laboral.

Esto implicaría que la docencia universitaria, con una adecuada formación en el uso de las tecnologías y de las prácticas educativas inclusivas, realice estrategias de mediación pedagógica que sean didácticas y que puedan ser accesibles para todo tipo de población, sin dejar de fomentar la criticidad, la transformación social, el aprendizaje significativo y demás aspectos que caracterizan la formación integral buscada por la Universidad Nacional.

\section{Conclusiones}

Sin duda, la docencia es un área donde nunca se deja de aprender, cada desafío es una oportunidad nueva para educarse; por ello, la inclusión debería empezar a significar una experiencia no solo para la docencia universitaria, sino para la Universidad Nacional como institución. Los avances que se han tenido son muchos y la labor que se realiza por lograr tener una sociedad inclusiva y justa es también evidente. Sin embargo, mientras haya una sola persona que esté siendo excluida, mucho no será suficiente.

Los estudios evidencian que no se está logrando un adecuado abordaje de la inclusión en la educación superior costarricense. También, se demuestra la existencia de mecanismos que apelan a que la inclusión sea una realidad en la Universidad Nacional, tanto desde la 
legislación nacional, como desde las decisiones políticas y de gestión del centro de estudios. Además, la institución cuenta con profesionales que podrían hacer que la inclusión para las personas con una discapacidad sea cada vez más una realidad y no una utopía.

Todo está en emplear los mecanismos con los que se cuenta y en no obviar el modelo pedagógico de la universidad y por supuesto, no olvidar cuáles fueron las bases sobre las que se asentó esta institución. Ellas se basaron en el fomento de una pedagogía esperanzadora y humanista, que contribuyera a las necesidades del país.

En este momento, la sociedad y los profesionales que egresan y que laboran en la Universidad Nacional requieren de una formación que les enseñe cómo enfrentar un desafío social como la inclusión, pues en el momento en que se responda a ese desafío serán menos las personas que se sientan excluidas de la sociedad solo por tener una deficiencia que no se le han otorgado los apoyos necesarios, sino que más bien se le han impuesto barreras.

De ahí la importancia de realizar procesos de reflexión sobre la propia praxis pedagógica, para poder conocer cuáles son los vacíos que se tienen en la propia formación profesional, pues en este caso, gracias a un proceso de autorreflexión, se logró visibilizar un vacío en la formación profesional de la docencia sobre prácticas educativas inclusivas.

De esta manera es posible ver cómo en la experiencia del campo (desde el aprendizaje autodidacta y empírico), es donde se están forjando las habilidades y conocimientos para saber cómo trabajar con las personas con discapacidad. Por esta razón, es necesario que el personal docente escriba sobre las experiencias vividas en su quehacer, pues esas sistematizaciones serán clave para desarrollar mejoras en la formación de formadores.

Por ello, es importante que los esfuerzos no surjan únicamente de la institución, sino también de quienes la componen, pues la universidad brinda las herramientas necesarias a los distintos profesionales para que desarrollen la capacidad y habilidad de crear y transformar. Si no son las mismas personas quienes formen parte de la universidad, promuevan y creen cambios, muy difícilmente se logrará responder al objetivo comúnactual : una sociedad justa, equitativa y libre. 


\section{Referencias}

Álvarez, P. (2012). Tutoría universitaria inclusiva: guía de buenas prácticas para la orientación de estudiantes con necesidades educativas especificas. Narcea Ediciones.

Consejo Nacional de Rehabilitación y Educación Especial. (2012). Política Nacional en Discapacidad 2011-2021. http://www.documentos.una. ac.cr/bitstream/handle/unadocs/4318/resumen_ponadis.pdf

Consejo Nacional de Personas con Discapacidad e Instituto Nacional de Estadística y Censos. (2019). Encuesta Nacional sobre Discapacidad 2018. http://www.inec.go.cr/sites/default/files/documetos-biblioteca-virtual/reenadis2018.pdf

Consejo Universitario. (2012). Lineamientos para la flexibilidad curricular en la Universidad Nacional. http://www.documentos.una. ac.cr/handle/unadocs/1117

Consejo Universitario. (2016). Políticas Institucionales. http://www. documentos.una.ac.cr/handle/unadocs/1149

Decreto 40955. Establecimiento de la inclusión y la accesibilidad en el Sistema Educativo Costarricense. Costa Rica, 13 de febrero del 2018. http://196.40.56.11/scij/Busqueda/Normativa/ Normas/nrm_norma.aspx?param1=NRM\&nValor1=1\&nValor $2=86181 \&$ nValor $3=111664 \&$ strTipM=FN

Echeita, G., Parrilla, A. y Carbonall, F. (2011). La educación especial a debate. Revista RUEDES de la Red Universitaria de Educación Especial, 1(1), 35-53. https://repositorio.uam.es/ handle/10486/668930

Ley $\mathrm{N}^{\circ} 7600$. Ley De Igualdad De Oportunidades Para Las Personas Con Discapacidad. San José, Costa Rica, 2 de mayo de 1996. http:// www.tse.go.cr/pdf/normativa/leyigualdaddeoportunidades.pdf

Ley $\mathrm{N}^{\circ}$ 7948. Convención Interamericana Para La Eliminación De Todas Las Formas De Discriminación Contra Las Personas Con Discapacidad. Ciudad de Guatemala, Guatemala, 8 de diciembre de 1999. https://www.oas.org/juridico/spanish/tratados/a-65.html

Ley N ${ }^{\circ}$ 8661. Convención Sobre Los Derechos De Las Personas Con Discapacidad. San José, Costa Rica, 19 de agosto del 2008. http:// www.documentos.una.ac.cr/handle/unadocs/4315

López, M. (2018). Fundamentos y Prácticas Inclusivas en el Proyecto Roma. Ediciones Morata. 
Madrigal, M. (2015). Redes de intermediación de empleo para personas con discapacidad. Organización Internacional del Trabajo.

Moliner, O., Sanahuja, A. y Benet, A. (2017). Prácticas inclusivas en el aula desde la investigación-acción. Universitat Jaume I. http:// repositori.uji.es/xmlui/bitstream/handle/10234/168902/s127. pdf? sequence $=1 \&$ isAllowed $=\mathrm{y}$

UniversidadNacional.(2012).ModeloPedagógico.https://www.una.ac.cr/ index.php/acerda-de/estrategia-institucional/2012-10-02-15-21-57

Velarde, V. (2012). Los modelos de la discapacidad: un recorrido histórico. Revista Empresa y Humanismo, 15(1), 115-136. https://dadun.unav.edu/bitstream/10171/29153/1/REYH\%2015-1-12Velarde\%20Lizama.pdf

Vijil, J. (2011). Hacia un nuevo modelo de formación: Programa de actualización de docentes universitarios de Ciencias Sociales. IHNCA-UCA. 
